\title{
Predictive value of standard serum markers for bone metastases in prostate cancer
}

\author{
Semra Ozdemir ${ }^{1^{*}}$ (D), Ahmet Reşit Ersay ${ }^{2}$ (D), Fulya Koc Ozturk ${ }^{1}$ (i) and Beril Su Ozdemir ${ }^{3}$ (D)
}

\begin{abstract}
Background: The early detection of bone metastases is very important in prostate cancer follow-up. This study aimed to compare conventional tumor markers, namely free prostate-specific antigen (free PSA), total prostate-specific antigen (total PSA), free PSA/total PSA ratio, alkaline phosphatase (ALP) values, Gleason scores and ${ }^{99} \mathrm{~m}$ Tc-MDP bone scintigraphy findings in the prediction of bone metastases in prostate cancer.

Methods: In total, 175 patients with prostate cancer who underwent whole-body bone scintigraphy were included in the study. All selected scintigraphic studies were reprocessed. Free PSA, total PSA, free PSA/total PSA ratio, alkaline phosphatase (ALP) values and Gleason scores of patients were recorded.

Results: The results of our study show that the presence of bone metastasis correlates very weakly with free PSA/ total PSA ratio $(r h o=0.179)$, weakly with total PSA $(r h o=0.318)$ and Gleason score $(r h o=0.382)$, moderately with ALP ( $r$ ho $=0.539$ ), free PSA (0.416). Only ALP variable had a diagnostic value and ALP cutoff value was $76.50 \mathrm{IU} / \mathrm{L}$, with $80 \%$ sensitivity and $82.1 \%$ specificity.

Conclusion: According to the results of our study; the free PSA, total PSA, free PSA/total PSA ratio and Gleason score values were not considered as a reliable parameter in the prostate cancer cases follow-up for bone metastasis development. Only ALP had a diagnostic value and ALP cutoff value was $76.50 \mathrm{IU} / \mathrm{L}$ with $80 \%$ sensitivity and $82.1 \%$ specificity in predicting bone metastases in prostate cancer.
\end{abstract}

Keywords: Bone scintigraphy, Free/total PSA, Prostate cancer

\section{Background}

Prostate cancer is the second-most commonly diagnosed cancer among men worldwide after lung cancer and ranks fifth for cancer-related mortality rates. Definitive prostate cancer diagnoses are made by biopsy. Patient selection for biopsy is important to prevent unnecessary diagnosis/treatment. The most important marker used to decide which patients should be considered for biopsy is the serum prostate-specific antigen (PSA) value [1-3]. However, PSA, which is a glycoprotein produced by the prostate tissue, increases not only in the case of prostate cancer but also in the case of a variety of benign diseases

\footnotetext{
*Correspondence: semozdemir@yahoo.com

${ }^{1}$ Department of Nuclear Medicine, Faculty of Medicine, Çanakkale

Onsekiz Mart University, 17100, Çanakkale, Turkey

Full list of author information is available at the end of the article
}

such as benign prostate hypertrophy $(\mathrm{BPH})$, prostatitis and urinary infections [4]. In addition, the specificity and positive predictive value of PSA, as a commonly used marker in prostate cancer screening, is low and an exact cutoff value has not yet been defined [5]. In clinical routine, the differentiation of $\mathrm{BPH}$ and prostate cancer in patients with PSA levels between $4.0 \mathrm{ng} / \mathrm{mL}$ and $10.0 \mathrm{ng} /$ $\mathrm{mL}$ is difficult $[6,7]$.

Due to these limitations, the use of different forms or kinetics of PSA has been recommended. However, recent studies have revealed that no biomarker alone is sufficient in diagnosis and a multivariable diagnostic approach is more eligible $[5,8,9]$. Nevertheless, due to some disadvantages of these markers, the free prostate-specific antigen/total prostate-specific antigen (free PSA/total PSA) ratio has become the most frequently used value in clinical practice $[9,10]$. Recently, it has been reported in the 
literature that the free PSA/total PSA ratio is more significant than PSA alone in differentiating prostate cancer and $\mathrm{BPH}$ in patients with serum total PSA levels between 4.0 and $10.0 \mathrm{ng} / \mathrm{mL}[11,12]$.

Prostate cancer mainly causes osteoblastic bone metastasis. The number of metastases in the bones is important in predicting the response to therapy and is generally associated with decreased survival rates [13]. Therefore, the early diagnosis of bone metastasis is substantial. Bone scintigraphy is frequently used as a non-invasive, inexpensive and easily accessible imaging method to detect bone metastasis. In addition, bone scintigraphy has been recommended in major urology guidelines as an imaging method for bone metastasis screening in medium-tohigh risk patient groups and symptomatic patients. PSA values, Gleason Scores and the clinical stage of the lesion have been known to affect the success rate of this method $[14,15]$. This study aimed to investigate the prognostic significance of the free PSA/total PSA ratio, which has been recently reported to be a more sensitive marker in detecting bone metastasis in prostate cancer.

\section{Methods}

\subsection{Patient group}

The patient group of this study consisted of a total of 175 caucasian (white) male patients with a mean age of $71.36 \pm 8.61$ years. The patients were selected retrospectively from those who had prostate cancer and had been sent to the Department of Nuclear Medicine of the Faculty of Medicine for a whole-body bone scintigraphy between 2016 and 2019. The patient population consisted of patients with various stages of prostate cancer who were referred to be further investigated to determined the presence of bone metastasis preoperatively or bone metastasis due to increased PSA at follow-up. The bone scintigraphy results were evaluated by two nuclear medicine specialists, and the number of bone metastases was recorded. In cases with more than 30 bone metastases, the number of metastases was recorded as 30. In addition to bone scintigraphy, the free PSA/total PSA and alkaline phosphatase (ALP) values measured over the last month were screened retrospectively and recorded along with the age, date of cancer diagnosis and Gleason scores of the patients.

Written informed consent was obtained from all patients. This retrospective study was approved by Canakkale Onsekiz Mart University Clinical Research Ethics Committee (Date: 07.05.2020, No:2020-07).

All patients read and signed the informed consent forms and were properly prepared for the procedure. For the bone scintigraphy, $15-30 \mathrm{mCi}$ (doses were adjusted to weight) Tc-99 $\mathrm{m}$-tagged diphosphonate compounds (MDP or HMDP) were injected into the patients intravenously. Approximately, 2-3 h after the injections were given, the whole-body images in the anterior and posterior projections and-if necessary-spot planar images of the suspicious regions were acquired. The whole-body bone scintigraphy imaging was realized with a dual-head gamma camera (Infinia; General Electric Medical Systems, Milwaukee, Wisconsin, USA) using an image matrix of $1024 \times 1024$ with a speed of $8 \mathrm{~cm} / \mathrm{min}$ for $10 \mathrm{~min}$ for each static. The prior surgery and trauma history of the patients were carefully noted. Asymmetric and multiple focal lesions, especially located in the axial skeleton, were evaluated as bone metastasis.

\subsection{Statistical analysis}

All data were analyzed using statistical package software SPSS (Statistical Package for Social Sciences) version 20.0. The descriptive data analysis included number, percentage, mean, standard deviation, minimum and maximum values. In the analytical data presentation, the conformity of the variables to the normal distribution was assessed using the Kolmogorov-Smirnov normality test, due to the fact that the number of patients included in the study was more than 50. The Pearson correlation test was used to analyzed the continuous variables with normal distribution, while the Spearman correlation test was used to analyze the continuous variables without normal distribution. The cutoff, sensitivity and specificity values were determined by the receiver-operating characteristic (ROC) curve analysis. A value of $p<0.05$ was considered statistically significant.

\section{Results}

The study population of the present study consisted of 175 male patients with prostate cancer who underwent whole-body bone scintigraphy to investigate the presence of bone metastasis. The mean age of the patients was $71.36 \pm 8.61$ years (minimum-maximum: 48.0-88.0). The mean free PSA and total PSA values of the patients were $19.21 \pm 43.02 \mathrm{ng} / \mathrm{ml}$ and $87.84 \pm 299.83 \mathrm{ng} / \mathrm{ml}$, respectively. The mean value of the free PSA/total PSA ratio was calculated as $0.026 \pm 0.731$. The mean ALP and Gleason score of the patients were $181.87 \pm 283.46 \mathrm{IU} / \mathrm{L}$ and $7.75 \pm 1.43$, respectively. The demographic and clinical characteristics of the case groups are summarized in Table 1 . The bone scintigraphy images of three prostate cancer patients at different stages are illustrated in Fig. 1.

When the free PSA, total PSA, free PSA/total PSA ratio, ALP values and Gleason scores were compared with number of bone metastases, the following were determined: 
Table 1 Demographic and clinical parameters

\begin{tabular}{lcrl}
\hline Clinical parameters & \multicolumn{3}{c}{ Patients cohort $(\boldsymbol{n}=\mathbf{1 7 5})$} \\
\cline { 2 - 4 } & Mean \pm SD & Median & Min.-Max. \\
\hline A.ge (years) & $71.36 \pm 8.61$ & 71.00 & $48-88$ \\
Free PSA $(\mathrm{ng} / \mathrm{ml})(n=95)$ & $19.21 \pm 43.02$ & 2.93 & $0-235$ \\
Total PSA $(\mathrm{ng} / \mathrm{ml})(n=175)$ & $87.84 \pm 299.83$ & 14.95 & $0-2934$ \\
Free/total PSA $(n=96)$ & $0.236 \pm 0.220$ & 0.18 & $0-1.67$ \\
Alkaline phosphatase(U/L) & $181.87 \pm 283.46$ & 91.00 & $12.80 \pm 1722$ \\
$\quad(n=57)$ & & & \\
Gleason score $(n=120)$ & $7.75 \pm 1.43$ & 8.00 & $4-10$ \\
$\quad \begin{array}{l}\text { Number of metastatic foci } \\
\quad(n=175)\end{array}$ & $9.68 \pm 12.13$ & 2.00 & $0-30$ \\
\hline
\end{tabular}

1. A very weak correlation was observed between the number of bone metastases lesions and the free PSA/ total PSA ratio $(\mathrm{rho}=0.179)$.

2. A weak correlation was found between the number of bone metastatic lesions and total PSA $(\mathrm{rho}=0.318)$ and Gleason scores ( $\mathrm{rho}=0.382$ ).

3. A moderate correlation was observed between the number of bone metastatic lesions and, ALP values $($ rho $=0.539)$ and free PSA (0.416) (Table 2).
The ROC curve of sensitivity versus the specificity of free PSA, total PSA, free PSA/total PSA ratio, ALP and Gleason scores was performed to detect bone metastases in prostate cancer. It was determined that only the ALP variable was statistically significant and had a high diagnostic value (AUC $=0.907, \mathrm{p}=0.004$ ). The ALP cutoff value was found to be $76.50 \mathrm{IU} / \mathrm{L}$, with a sensitivity rate of $80 \%$ and a specificity rate of $82.1 \%$. The obtained results also showed that there was a moderate correlation between bone metastasis and ALP values. On the other hand, according to the results, the free PSA (AUC $=0.657, \mathrm{p}=0.269)$, total PSA $\quad(\mathrm{AUC}=0.661$, $\mathrm{p}=0.259)$, free PSA/total PSA ratio $(\mathrm{AUC}=0.521$, $\mathrm{p}=0.880)$ and Gleason score $(\mathrm{AUC}=0.529, \mathrm{p}=0.841)$ values were not considered as reliable parameters for the detection of bone metastasis in prostate cancer cases. (Fig. 2, Table 3).

\section{Discussion}

Serum PSA is currently the most commonly used marker in the diagnosis and follow-up of prostate cancer. The use of PSA for prostate cancer screening was approved in 1994 by the US Food and Drug Administration [16, 17]. However, PSA is not a cancer-specific marker, leading to unnecessary biopsies, especially in the range of

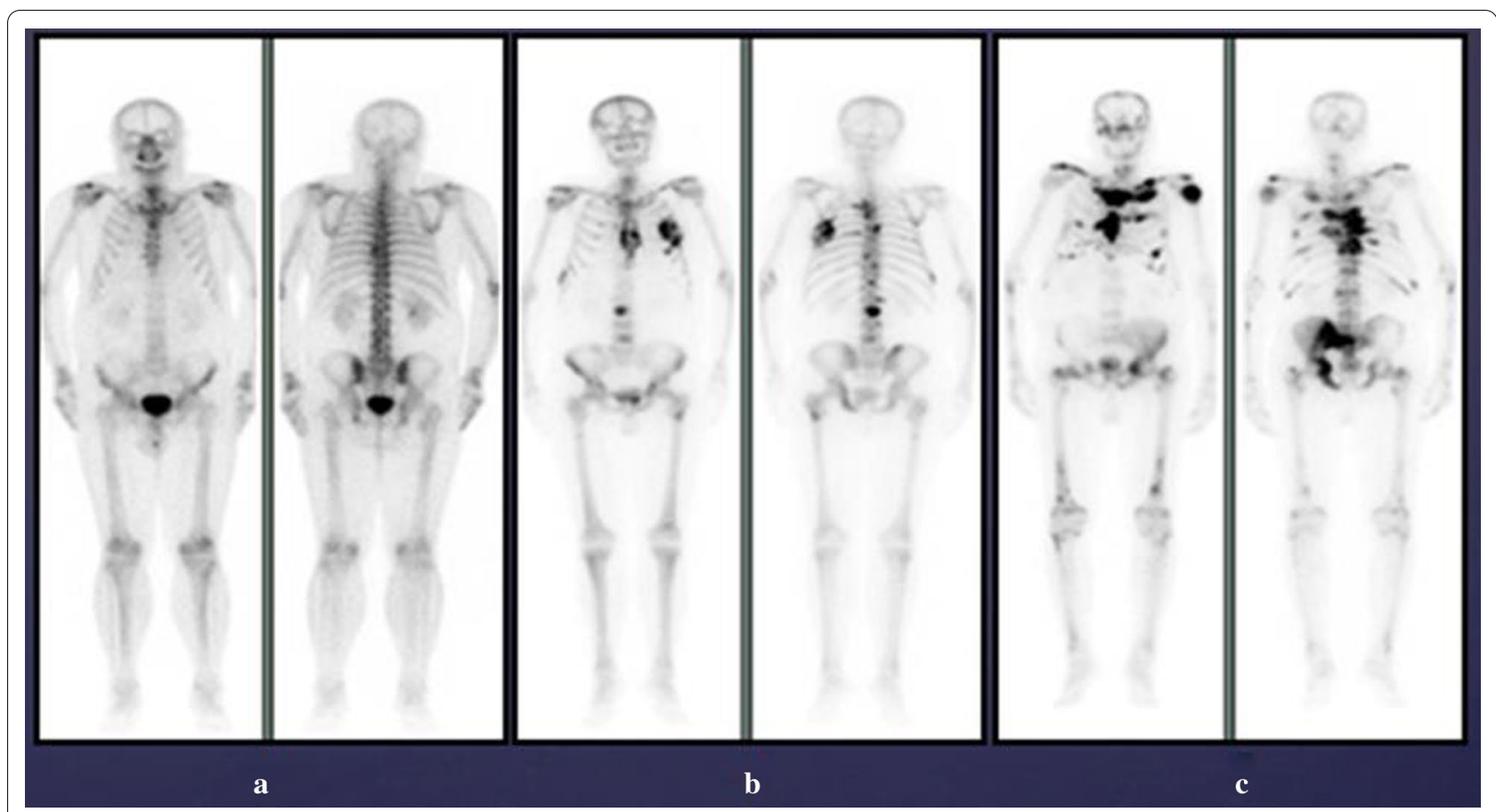

Fig. 1 Bone scintigraphy images of three prostate cancer patients at different stages. a: An example patient without bone metastases; free PSA: $2.53 \mathrm{ng} / \mathrm{ml}$, total PSA: $17.54 \mathrm{ng} / \mathrm{ml}$, free/total PSA ratio: 0.144, ALP: $43 \mathrm{U} / \mathrm{L}$, b: An example patient with numerous metastases; free PSA: $3.11 \mathrm{ng} /$ ml, total PSA:18.30 ng/ml, free/total PSA ratio: 0.169, ALP: 114 U/L. C: An example patient with multiple metastases; free PSA: $12.90 \mathrm{ng} / \mathrm{ml}$, total PSA:121.20 ng/ml, free/total PSA ratio: 0.106, ALP: $109 \mathrm{U} / \mathrm{L}$ 
Table 2 Spearman's correlation coefficients of bone metastases lesion number and some laboratory parameters.

\begin{tabular}{ll}
\hline Parameters & $\begin{array}{l}\text { Bone metastases } \\
\text { lesion number }\end{array}$ \\
\hline Free PSA $(n=96)$ & 0.416 \\
Spearman's correlation rho & $<0.001$ \\
$p$ & \\
Total PSA $(n=175)$ & 0.318 \\
Spearman's correlation rho & $<0.001$ \\
$P$ & \\
Free/total PSA $(n=96)$ & 0.179 \\
Spearman's correlation rho & 0.082 \\
$p$ & \\
ALP $(n=57)$ & 0.539 \\
Spearman's correlation rho & $<0.001$ \\
$p$ & \\
Gleason Score $(n=120)$ & 0.382 \\
Spearman's correlation rho & $<0.001$ \\
$p$ & \\
\hline
\end{tabular}

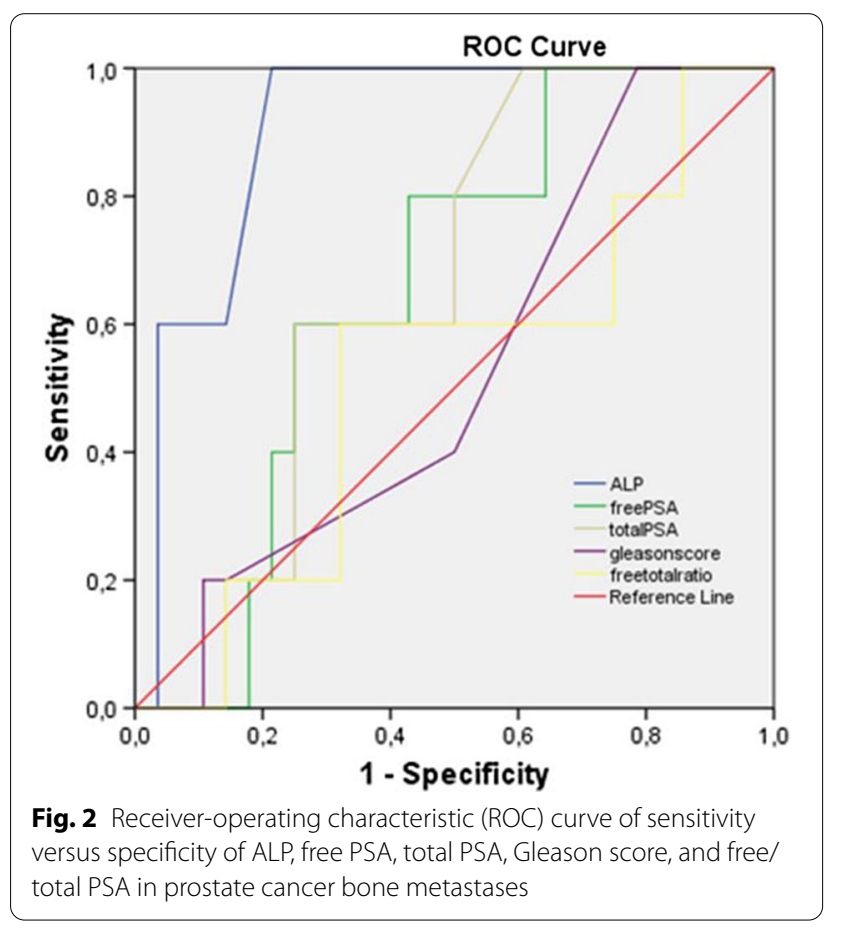

$4.0-10.0 \mathrm{ng} / \mathrm{mL}$, which is defined as the gray zone. In this range, the most commonly used parameter to increase the specificity of PSA is the free PSA/total PSA ratio. While $5 \%$ of serum PSA is found to be free, this level decreases in patients with prostate cancer. Therefore, free PSA measurement is used to increase the sensitivity of cancer detection in patients with normal PSA values and increase specificity in patients with high total PSA (2.5$10 \mathrm{ng} / \mathrm{mL})[18,19]$.

An increasing number of reports published in recent years have demonstrated that the free PSA/total PSA ratio can be used for determining the necessity of biopsy in prostate cancer diagnoses $[12,20]$. Erol et al. conducted a study on 4955 patients with total PSA values between 4 and $10 \mathrm{ng} / \mathrm{ml}$ and determined that a free PSA/ total PSA ratio of $<0,10$ had the highest specificity, while a ratio of $>0,30$ had the highest sensitivity in prostate cancer diagnosis. In addition, this study demonstrated that the free PSA/total PSA ratio varied according to age [21].

Catalona et al. reported that the free PSA/total PSA ratio significantly decreased in the presence of aggressive prostate cancer (Gleason score $\geq 7$, presence of metastasis). In addition, they determined that the 0,25 cutoff value for the free PSA/total PSA ratio would have a sensitivity of $95 \%$ in prostate cancer diagnosis and therefore prevent $20 \%$ of unnecessary biopsies [22]. The National Comprehensive Cancer Network (NCCN) guideline recommends using the free PSA/total PSA ratio for TRUSguided prostate biopsy decisions in patients with no prior biopsy history, those with a PSA value of $>3 \mathrm{~g} / \mathrm{ml}$ or high-risk patients with prior negative biopsy results [23]. Similarly, The European Urology Association (EUA) guideline reported that the free PSA/total PSA ratio could be used to avoid unnecessary biopsies in patients with total PSA values between 2 and $10 \mathrm{ng} / \mathrm{ml}$ [24]. Likewise, Bjork et al. determined a low free PSA/total PSA ratio in male patients with prostate cancer [25]. The free PSA/total PSA ratio, calculated using simultaneous immune determination of both free PSA and total PSA, has been reported to yield better results than PSA or its derivations alone in prostate cancer diagnosis. This ratio

Table 3 Sensitivity and specificity percentage of ALP, free PSA, total PSA, Gleason score, and free/total PSA values

\begin{tabular}{lllllll}
\hline & & Free PSA $\boldsymbol{n}=\mathbf{9 6}$ & $\begin{array}{l}\text { Total PSA } \\
\boldsymbol{n}=\mathbf{1 7 5}\end{array}$ & $\begin{array}{l}\text { Free/total PSA } \\
\boldsymbol{n}=\mathbf{9 6}\end{array}$ & ALP $\boldsymbol{n}=\mathbf{5 7}$ & Gleason $\boldsymbol{n}=\mathbf{1 2 0}$ \\
\hline Bone metastases number & $P$ & 0.269 & 0.259 & 0.880 & 0.004 & 0.841 \\
& AUC & 0.343 & 0.339 & 0.479 & 0.093 & 0.471 \\
& Cutoff & 5.77 & 25.1 & 0,15 & 76.5 & 8.5 \\
& Sensitivity \% & 60 & 60 & 60 & 80 & 40 \\
& Specificity \% & 60.7 & 60.7 & 60.7 & 82.1 & 50 \\
\hline
\end{tabular}


is recommended to be used especially in cases where total PSA levels are low.

On the other hand, various studies have reported that the free PSA/total PSA ratio is not as significant as expected. For example, Agnihotri $\mathrm{S}$ et al. concluded that the value of free PSA/total PSA in symptomatic males and found a very limited value to improve specificity of total PSA [26]. Moreover, Huyghe E et al. claimed that the calculation of the free PSA/ total PSA ratio did not appear to provide any decisional criteria in favor of radical prostatectomy [27].

Although ${ }^{68}$ Ga-PSMA PET-CT imaging is in the gold standard position as it can evaluate the whole body (bone, soft tissue, lymph node, etc.) in the follow-up of prostate cancer, it is expensive and not always accessible [28]. ${ }^{99 \mathrm{~m}} \mathrm{Tc}-\mathrm{MDP}$ whole-body bone scintigraphy is still a highly sensitive imaging method in cases that only bone metastases are investigated due to its availability and low cost [29]. A meta-analysis reported a patient-level sensitivity rate of $79 \%$ and a specificity rate of $82 \%$ and a lesion-level sensitivity of $59 \%$ and specificity of $75 \%$ [30]. The correlation between bone metastasis and PSA level, ALP level and Gleason score has been previously investigated, and the presence of correlation at different levels has been mentioned in the literature [31-33]. Some studies have recommended the use of bone scans in symptomatic patients or if the alkaline phosphatase levels of the patients are $>90 \mathrm{U} / 1$ [34]. Singh et al. claim that there was a PSA cutoff of $10 \mathrm{ng} / \mathrm{ml}$ for negative bone scans and a correlation between PSA and the presence and number of metastases [35]. On the other hand, a recent study suggested that the staging of bone scans may not be performed in patients with a PSA level of $<20 \mathrm{ng} / \mathrm{mL}$ [36]. However, no clear results were obtained in these studies that focused on determining a cutoff value for the aforementioned parameters in detecting bone metastasis.

The objective of the present study was to compare conventional tumor markers, namely free PSA, total PSA, free PSA/ total PSA ratio, ALP values, Gleason scores and $99 \mathrm{~m} \mathrm{Tc}$-MDP bone scintigraphy findings in terms of the detection of bone metastasis. According to the obtained results, a very weak correlation was observed between the number of bone metastases lesions and the free PSA/total PSA ratio $($ rho $=0.179)$; a weak correlation was found between the number of bone metastatic lesions and total PSA $(\mathrm{rho}=0.318)$ and Gleason scores $($ rho $=0.382)$; a moderate correlation was observed between the number of bone metastatic lesions and, ALP values $($ rho $=0.539)$ and free PSA (0.416). As bone scintigraphy is a highly sensitive technique used to detect bone metastases, the results of this study led to the conclusion that free PSA, total PSA, free PSA/total PSA ratio and Gleason score values were not reliable parameters in the follow-up of bone metastases in prostate cancer cases. On the other hand, the $76.50 \mathrm{IU} / \mathrm{L}$ cutoff value for ALP values showed a sensitivity ratio of $80.0 \%$ and a specificity ratio of $82.1 \%$ for the prediction of bone metastases.

\section{Conclusion}

The results of this study showed that the free PSA, total PSA, free PSA/total PSA ratio and Gleason score values were not reliable parameters for determining the bone metastases of prostate cancer. It was determined that only the ALP variable had a diagnostic value in predicting bone metastases in prostate cancer. On the other hand, it was thought that routine control and patient complaints maintained their importance in the follow-up of bone metastases.

\section{Abbreviations}

ALP: Alkaline phosphatase; BPH: Benign prostate hypertrophy; Free PSA/ total PSA: Free prostate-specific antigen/total prostate-specific antigen; PSA: Prostate-specific antigen.

\section{Acknowledgements \\ The authors thank the family members who agreed to participate in the presented results.}

\section{Authors' contributions}

SO., ARE. contributed to concept and design; SO. contributed to supervision; FKO. contributed to material; BSO., FKO contributed to data collection and/or processing; SO., ARE. contributed to analysis and/or interpretation; SO., BSO. contributed to literature search; SO contributed to writing manuscript. All authors read and approved the final manuscript.

\section{Funding}

No funding was obtained for this study.

\section{Availability of data and materials}

All data generated or analyzed during this study are included in this published article.

\section{Declarations}

\section{Ethics approval and consent to participate}

Ethics committee approval was received for this study from the Canakkale Onsekiz Mart University Clinical Research Ethics Committee local ethics committee (Date: 07.05.2020, No:2020-07).

\section{Consent for publication}

Not Applicable.

\section{Competing interests}

The authors declare that they have no competing interests.

\section{Informed consent}

Informed written consent was obtained from all patients.

\section{Author details}

${ }^{1}$ Department of Nuclear Medicine, Faculty of Medicine, Çanakkale Onsekiz Mart University, 17100, Çanakkale, Turkey. ${ }^{2}$ Department of Urology, Faculty of Medicine, Çanakkale Onsekiz Mart University, 17100, Çanakkale, Turkey. ${ }^{3}$ Department of Research and Development, Santa Farma Pharmaceuticals 41000, Kocaeli, Turkey. 
Received: 3 November 2020 Accepted: 29 April 2021

Published online: 11 May 2021

\section{References}

1. Abdollah F, Dalela D, Haffner MC, Culig Z, Schalken J (2015) The role of biomarkers and genetics in the diagnosis of prostate cancer. Eur Urol Focus 1:99-108

2. Moradi A, Srinivasan S, Clements J, Batra J (2019) Beyond the biomarker role: prostate-specific antigen (PSA) in the prostate cancer microenvironment. Cancer Metastasis Rev 38:333-346

3. Nevo A, Navaratnam A, Andrews P (2020) Prostate cancer and the role of biomarkers. Abdom Radiol (NY) 45(7):2121-2132

4. Bernal-Soriano MC, Parker LA, López-Garrigos M, Hernández-Aguado I, Caballero-Romeu JP, Gómez-Pérez L et al (2019) Factors associated with false negative and false positive results of prostate-specific antigen (PSA) and the impact on patient health: cohort study protocol. Med (Baltimore) 98(40):e17451. https://doi.org/10.1097/MD.0000000000017451

5. Horwich A, Parker C, de Reijke T, Kataja V (2013) ESMO Guidelines Working group. Prostate cancer: ESMO clinical practice guidelines for diagnosis, treatment and follow-up. Ann Oncol 24:106-114. https://doi.org/10. 1093/annonc/mdt208

6. Carroll PR, Vickers AJ (2014) Point/counterpoint: early detection of prostate cancer: do the benefits outweigh the consequences? J Natl Compr Canc Netw 12:768-771

7. Kumar N, Yadav S, Kumar S, Saurav K, Prasad V, Vasudeva P (2019) Comparison of percentage free PSA, MRI and Ga PSMA PET scan for diagnosing cancer prostate in men with PSA between 4 and $20 \mathrm{ng} / \mathrm{ml}$. Indian J Urol 35:202-207

8. Verma A, St Onge J, Dhillon K, Chorneyko A (2014) PSA density improves prediction of prostate cancer. Can J Urol 21:7312-7321

9. Nordström T, Akre O, Aly M, Grönberg H, Eklund M (2018) Prostate-specific antigen (PSA) density in the diagnostic algorithm of prostate cancer. Prostate Cancer Prostatic Dis 21:57-63

10. Wu ZY, Yang C, Luo J, Deng SL, Wu B, Chen M (2019) Establishment of reference intervals for serum [-2]proPSA (p2PSA), \%p2PSA and prostate health index in healthy men. Onco Targets Ther 13(12):6453-6460

11. Ito K, Yamamoto T, Ohi M, Kurokawa K, Suzuki K, Yamanaka H (2003) Free/ total PSA ratio is a powerful predictor of future prostate cancer morbidity in men with initial PSA levels of 4.1 to $10.0 \mathrm{ng} / \mathrm{mL}$. Urology 61:760-764

12. Caliskan S (2017) Diagnostic efficacy of free prostate-specific antigen/ total prostate-specific antigen ratio for the diagnosis of prostate cancer in low concentration $(<4 \mathrm{ng} / \mathrm{ml})$ and intermediate levels of total prostatespecific antigen (4.01-10.0 ng/ml). J Cancer Res Ther 13:279-283

13. Koizumi M, Motegi K, Koyama M, Terauchi T, Yuasa T, Yonese J (2017) Diagnostic performance of a computer-assisted diagnosis system for bone scintigraphy of newly developed skeletal metastasis in prostate cancer patients: search for low-sensitivity subgroups. Ann Nucl Med 31:521-528

14. Thomsen FB, Westerberg M, Garmo H, Robinson D, Holmberg L, Ulmert HD et al (2020) Prediction of metastatic prostate cancer by prostate-specific antigen in combination with T stage and Gleason Grade: nationwide, population-based register study. PLoS ONE 15(1):e0228447. https://doi. org/10.1371/journal.pone.0228447

15. Valero J, Peleteiro P, Henríquez I, Conde A, Piquer T, Lozano A et al (2020) Age gleason score and PSA are important prognostic factors for survival in metastatic castration-resistant prostate cancer results of The Uroncor group (Uro-Oncological Tumors) of the Spanish society of radiation oncology (SEOR). Clin Transl Oncol. https://doi.org/10.1007/ s12094-019-02274-W

16. Kouriefs C, Sahoyl M, Grange P, Muir G (2009) Prostate specific antigen through the years. Arch Ital Urol Androl 81:195-198

17. Reed AB, Parekh DJ (2010) Biomarkers for prostate cancer detection. Expert Rev Anticancer Ther 10:103-114. https://doi.org/10.1586/era.09. 168

18. Vezyraki P, Vlachaki A, Baltogiannis D, Batistatou A, Tsampalas S, Simos V (2019) Impact of total PSA and percent free PSA in the differentiation of prostate disease: a retrospective comparative study implicating neoplastic and non-neoplastic entities. J BUON 24:2107-2113

19. Esfahani M, Ataei N, Panjehpour M (2015) Biomarkers for evaluation of prostate cancer prognosis. Asian Pac J Cancer Prev 16:2601-2611

20. Tanguay S, Bégin LR, Elhilali MM, Behlouli H, Karakiewicz PI, Aprikian AG (2002) Comparative evaluation of total PSA, free/total PSA, and complexed PSA in prostate cancer detection. Urology 59:261-265

21. Erol B, Gulpinar MT, Bozdogan G, Ozkanli S, Onem K, Mungan G et al (2014) The cutoff level of free/total prostate specific antigen ( $f / t$ PSA) ratios in the diagnosis of prostate cancer: a validation study on a Turkish patient population in different age categories. Kaohsiung J Med Sci 30:545-550. https://doi.org/10.1016/j.kjms.2014.03.008

22. Catalona WJ, Partin AW, Slawin KM, Naughton CK, Brawer MK, Flanigan RC et al (2000) Percentage of free PSA in black versus white men for detection and staging of prostate cancer: a prospective multicenter clinical trial. Urology 55:372-376

23. - -NCCN Clinical Practice Guidelines in Oncology (NCCN Guidelines) Prostate Cancer. Versión 2.2019; 2019. NCCN org.

24. Mottet N, Bellmunt J, Bolla M, Briers E, Cumberbatch MG et al (2017) EAUESTRO-SIOG guidelines on prostate cancer. part 1: screening, diagnosis, and local treatment with curative intent. Eur Urol 71:618-629

25. Björk T, Lilja H, Christensson A (1999) The prognostic value of different forms of prostate specific antigen and their ratios in patients with prostate cancer. BJU Int 84:1021-1027

26. Agnihotri S, Mittal RD, Ahmad S, Mandhani A (2014) Free to total serum prostate specific antigen ratio in symptomatic men does not help in differentiating benign from malignant disease of the prostate. Indian J Urol 30:28-32

27. Huyghe E, Soulie M, Tollon C, Escourrou G, Pontonnier F, Plante P (1999) Value of free PSA/total PSA ratio in therapeutic decisions in the case of a single positive biopsy of the prostate. Prog Urol 9:281-287

28. Lenzo NP, Meyrick D, Turner JH (2018) Review of gallium-68 PSMA PET/CT imaging in the management of prostate cancer. Diagnost (Basel) 8(1):16

29. Sevcenco S, Grubmüller B, Sonneck-Koenne C, Ahmadi Y, Knoll P, Floth A et al (2019) Bone scintigraphy in staging of newly diagnosed prostate cancer in regard of different risk groups. Asia Ocean J Nucl Med Biol Spring 7:149-152

30. Suh $\mathrm{CH}$, Shinagare $\mathrm{AB}$, Westenfield $\mathrm{AM}$, Ramaiya $\mathrm{NH}$, Van den Abbeele AD, Kim KW (2018) Yield of bone scintigraphy for the detection of metastatic disease in treatment-naive prostate cancer: a systematic review and meta-analysis. Clin Radiol 73:158-167

31. Wei RJ, Li TY, Yang XC, Jia N, Yang XL, Song HB (2016) Serum levels of PSA, ALP, ICTP, and BSP in prostate cancer patients and the significance of ROC curve in the diagnosis of prostate cancer bone metastases. Genet Mol Res. https://doi.org/10.4238/gmr.15027707

32. Ozu C, Nakashima J, Horiguchi Y, Oya M, Ohigashi T, Murai M (2008) Prediction of bone metastases by combination of tartrate-resistant acid phosphatase, alkaline phosphatase and prostate specific antigen in patients with prostate cancer. Int J Urol 15:419-422

33. Shepherd KL, Cool P, Cribb G (2018) Prognostic indicators of outcome for patients with skeletal metastases from carcinoma of the prostate. Bone Joint J 100:1647-1654

34. Moslehi M, Cheki M, Salehi-Marzijarani M, Amuchastegui T, Gholamrezanezhad A (2013) Predictors of bone metastasis in pre-treatment staging of asymptomatic treatment-naïve patients with prostate cancer. Rev Esp Med Nucl Imagen Mol 32:286-289

35. Singh OP, Yogi V, Redhu P, Ghori HU, Pareek A, Lal N (2019) Role of serum prostate-specific antigen as predictor for bone metastases in newly diagnosed prostate cancer. J Cancer Res Ther 15:39-S41

36. Al-Ghazo MA, Ghalayini IF, Al-Azab RS, Bani-Hani I, Barham A, Haddad Y (2010) Do all patients with newly diagnosed prostate cancer need staging radionuclide bone scan? A retrospective study. Int Braz J Urol 36:685-691 